Published in final edited form as:

Inorg Chem. 2006 September 4; 45(18): 7050-7052.

\title{
Coordination of Diatomic Ligands to Heme: Simply CO
}

\author{
Nathan J. Silvernail ${ }^{\dagger}$, Bruce C. NolI ${ }^{\dagger}$, Charles E. Schulz ${ }^{*}, \ddagger$, and W. Robert Scheidt ${ }^{\star}, \dagger$ \\ Department of Chemistry and Biochemistry, University of Notre Dame, Notre Dame, Indiana 46556 \\ Department of Physics, Knox College, Galesburg, Illinois 61401
}

\begin{abstract}
The synthesis and molecular structures of three iron(II) porphyrinates with only $\mathrm{CO}$ as the axial ligand(s) are reported. Two five-coordinate $[\mathrm{Fe}(\mathrm{OEP})(\mathrm{CO})]$ derivatives have $\mathrm{Fe}-\mathrm{C}=1.7077$ (13) and $1.7140(10) \AA$, much shorter than those of six-coordinate $[\mathrm{Fe}(\mathrm{OEP})(\mathrm{Im})(\mathrm{CO})]$, although $v_{\mathrm{C}-\mathrm{O}}$ is 1944$1948 \mathrm{~cm}^{-1}$. The six-coordinate species $\left[\mathrm{Fe}(\mathrm{OEP})(\mathrm{CO})_{2}\right]$ has also been studied. The competition for $\pi$-back-bonding of two CO ligands leads to $\mathrm{Fe}-\mathrm{C}$ distances of 1.8558 (10) $\AA$ and $v_{\mathrm{C}-\mathrm{O}}$ is increased to $2021 \mathrm{~cm}^{-1}$. The Mössbauer spectrum has a quadrupole splitting constant of $0 \mathrm{~mm} / \mathrm{s}$ at $4.2 \mathrm{~K}$, indicating high electronic symmetry.
\end{abstract}

Many heme-based sensing proteins that use/detect the diatomic ligands $\mathrm{O}_{2}, \mathrm{CO}$, or $\mathrm{NO}$ are known. ${ }^{1-4}$ These sensing proteins rely on variations in coordination number and the character of their axial ligands to induce conformational changes leading to protein activation. ${ }^{1}$ Effects involving the trans ligand (or lack thereof) on binding diatomic molecules are important in elucidating the mechanism of small-molecule-sensing proteins. In order to achieve a better understanding of the heme interactions involved with diatomic ligand sensing as well as broadly extending our understanding of their electronic and molecular structure, we have been investigating heme/diatomic ligand interactions.

We report for the first time the solid-state syntheses and structural characterization of unambiguously five-coordinate and six-coordinate heme $\mathrm{CO}$ complexes: $[\mathrm{Fe}(\mathrm{OEP})(\mathrm{CO})],[\mathrm{Fe}$ $(\mathrm{OEP})(\mathrm{CO})] . \mathrm{C}_{6} \mathrm{H}_{6}$, and $\left[\mathrm{Fe}(\mathrm{OEP})(\mathrm{CO})_{2}\right] .{ }^{5,6}$ Although these species have been previously reported in solution and $\mathrm{CO}$ binding constants determined for three different porphyrins, ${ }^{7}$ we now demonstrate the structural effects of changing coordination environments. We compare the five-coordinate structures with those of several other diatomic ligand heme complexes, examine effects of the addition of a sixth ligand and note structural differences.

These adducts may elucidate important coordination chemistry features involving ligand loss, ligand switching, and ligand photolability. CO photolysis of six-coordinate heme carbonyl derivatives has been a mainstay in biophysical investigations, ${ }^{8}$ and is very efficient with quantum yields nearing unity. The suggestion that five-coordinate heme carbonyls are less photolabile than their six-coordinate counterparts ${ }^{9}$ generates important questions about structural differences.

We first consider the vibrational properties of five-coordinate $[\mathrm{Fe}(\mathrm{OEP})(\mathrm{CO})] \cdot v_{\mathrm{C}-\mathrm{O}}$ in unperturbed imidazole-ligated hemes is typically near $1970 \mathrm{~cm}^{-1}$, but solid-state environmental factors can lead to variation in $v_{\mathrm{C}-\mathrm{O}}$ in the range of 1926 to $2000 \mathrm{~cm}^{-1.10,11}$ Thus, it is perhaps surprising to find that $v_{\mathrm{C}-\mathrm{O}}$ in $[\mathrm{Fe}(\mathrm{OEP})(\mathrm{CO})]$ and $[\mathrm{Fe}(\mathrm{OEP})(\mathrm{CO})] \cdot \mathrm{C}_{6} \mathrm{H}_{6}$ is

* To whom correspondence should be addressed: E-mail: Scheidt.1@nd.edu.

†University of Notre Dame.

\$nox College.

Supporting Information Available: Syntheses, figures and crystallographic data are available (including CIF files). This material is available free of charge via the Internet at http://pubs.acs.org. 
well within this range at $1944 \mathrm{~cm}^{-1}$ and $1948 \mathrm{~cm}^{-1}$, respectively (Nujol mull). These values might suggest that the iron center is involved in a significant intermolecular interaction to form a pseudo six-coordinate complex, but this is not correct (vide infra).

The molecular structures of the two crystalline $[\mathrm{Fe}(\mathrm{OEP})(\mathrm{CO})]$ complexes are illustrated in Figure 1 (and Fig. S1). There are strong similarities as well as differences in their structures: the iron out-of-plane displacements are similar at $0.20 \AA$ or $0.22 \AA$, the off-axis tilt of the $\mathrm{Fe}-$ $\mathrm{C}$ vector is $3.8^{\circ}$ or $2.4^{\circ}$, and the $\mathrm{Fe}-\mathrm{C}-\mathrm{O}$ bond angle is $177.20(8)^{\circ}$ in both. Both forms show some ring-ring interactions although these lead to some strong differences as well. Figure $2 \mathrm{a}$ shows the pairwise interactions in $[\mathrm{Fe}(\mathrm{OEP})(\mathrm{CO})]$, while the equivalent packing in the benzene solvate is shown in Figure 2b. In both cases, there is inversion symmetry; metrical information is given in the figure caption. The inter-ring packing pattern in the unsolvated form of [Fe $(\mathrm{OEP})(\mathrm{CO})]$ (Figure 2a) is similar to that of both crystalline forms of $[\mathrm{Fe}(\mathrm{OEP})(\mathrm{NO})]$ for which little por-phyrin overlap is observed. ${ }^{12}$ The closest intermolecular contact to the iron in $[\mathrm{Fe}$ $(\mathrm{OEP})(\mathrm{CO})]$ is $3.12 \AA$ whereas in $[\mathrm{Fe}(\mathrm{OEP})(\mathrm{CO})] \cdot \mathrm{C}_{6} \mathrm{H}_{6}$ the closest contact to iron is $3.58 \AA$. In the two $[\mathrm{Fe}(\mathrm{OEP})(\mathrm{NO})]^{+}$structures (isoelectronic to the CO's), however, there is a large porphyrin ring overlap with the $\pi$-system of an adjacent molecule acting as a pseudo sixth ligand. ${ }^{13}$ The tighter interactions in $[\mathrm{Fe}(\mathrm{OEP})(\mathrm{CO})] \cdot \mathrm{C}_{6} \mathrm{H}_{6}$ (Figure $2 \mathrm{~b}$ ) are comparable although slightly larger than in the isoelectronic $[\mathrm{Fe}(\mathrm{OEP})(\mathrm{NO})]^{+}$complexes. The porphyrin core conformation in $[\mathrm{Fe}(\mathrm{OEP})(\mathrm{CO})]$ is nearly planar whereas that in $[\mathrm{Fe}(\mathrm{OEP})(\mathrm{CO})] \cdot \mathrm{C}_{6} \mathrm{H}_{6}$ displays modest core ruffling. The average equatorial $\mathrm{Fe}-\mathrm{Np}$ distances are 1.988 (2) $\AA$ and 1.984(3) $\AA$ for the unsolvated and solvated forms. The very short $\mathrm{Fe}-\mathrm{N}_{\mathrm{p}}$ bond distances reflect the strong bonding interaction and low-spin state of iron. Significantly, the axial ligand-induced equatorial $\left(\mathrm{Fe}-\mathrm{N}_{\mathrm{p}}\right)$ bond distance differences observed in five-coordinate [Fe(por)(NO)] derivatives $^{12}$ are not observed; individual $\mathrm{Fe}-\mathrm{Np}$ distances are tightly clustered.

The short axial $\mathrm{Fe}-\mathrm{C}$ distance of $1.7140(11) \AA$ in $[\mathrm{Fe}(\mathrm{OEP})(\mathrm{CO})]$ is shorter than those of the six-coordinate imidazole adducts by $\sim 0.03-0.06 \AA .10,14$ This might suggest stronger $\mathrm{Fe} \rightarrow \mathrm{C}$ $\pi$-back-donation, but the relatively normal $v_{\mathrm{C}-\mathrm{O}}$ value suggests that the $\mathrm{Fe} \pi$-donation into the $\mathrm{CO} \pi^{*}$ orbitals is similar to that of the six-coordinate species. This would then suggest that it is the $\sigma$-bonding component that leads to the shorter $\mathrm{Fe}-\mathrm{C}$ bond distance. The distance in $[\mathrm{Fe}$ $(\mathrm{OEP})(\mathrm{CO})] . \mathrm{C}_{6} \mathrm{H}_{6}$ is 1.7077 (13) $\AA$; the possibly slightly shorter $\mathrm{Fe}-\mathrm{C}$ distance is not consistent with the $4 \mathrm{~cm}^{-1}$ increase in $v_{\mathrm{C}-\mathrm{O}}$, again suggesting the importance of $\sigma$-bonding. We thus conclude that differences in the $\sigma$-bonding component of $\mathrm{Fe}-\mathrm{C}$ have no or modest effects on $v_{\mathrm{C}-\mathrm{O}}$. As we have noted for a different series of six-coordinate carbonyl derivatives, ${ }^{10}$ there is a strong correlation between $v_{\mathrm{C}-\mathrm{O}}$ and the $\mathrm{Fe}-\mathrm{C} / \mathrm{C}-\mathrm{O}$ bond lengths, this question is being examined in more detail for OEP derivatives.

The structures of other $[\mathrm{Fe}(\mathrm{OEP})(\mathrm{XY})]^{+, 0}$ adducts $(\mathrm{XY}=\mathrm{NO}$ or $\mathrm{CS})$ are available for comparison. Structural parameters for five- and six-coordinate species are listed in Table 1. The iron out-of-plane displacement of [Fe(OEP)-(CO)] is seen to be at the low end of the range. In all cases, the addition of a sixth ligand leads to a decreased iron atom displacement and in most cases to an increase in the length of the $\mathrm{Fe}-\mathrm{X}(\mathrm{XY})$ bond. Although this increase is never large, the $\mathrm{COs}$ are the system that experiences the largest increase in $\mathrm{Fe}-\mathrm{C}$ bond distance (from $1.7140(11) \AA$ to $1.7733(12) \AA$ in $[\mathrm{Fe}(\mathrm{OEP})(\mathrm{CO})(1-\mathrm{MeIm})] .{ }^{14}$ Interestingly, the $\mathrm{Fe}-\mathrm{C}$ bond would appear to have changed minimally when the trans ligand is the weakly coordinating tetrahydrofuran ligand in $[\mathrm{Fe}(\mathrm{Deut})(\mathrm{CO})(\mathrm{THF})]$. Unfortunately, the relatively low precision of this structure (from $\sim 25$ years ago) is not adequate to comment on further, but clearly merits further attention.

Although solutions of $\mathrm{Fe}(\mathrm{OEP})$ and $\mathrm{CO}$ will always be mixtures of mono- and bis-CO species, crystallization experiments (see SI) at low temperature reproducibly afford crystals of [Fe $\left.(\mathrm{OEP})(\mathrm{CO})_{2}\right]$. Analysis of crystals of $\left[\mathrm{Fe}(\mathrm{OEP})(\mathrm{CO})_{2}\right]$ gave the structure displayed in Figure 
3; the complex has a required inversion center. As might be expected, the competition from two COs for $\pi$-backbonding from the central iron leads to increased $\mathrm{Fe}-\mathrm{C}$ bond distances of 1.8558 (10) $\AA$, which is also consistent with the observed asymmetric stretch of $2021 \mathrm{~cm}^{-1}$. The CO ligands are tilted off-axis as shown with other metrical information given in Figure 3. Perhaps as a consequence of the competition for bonding with two $\mathrm{CO}$ ligands, the equatorial $\mathrm{Fe}-\mathrm{N}_{\mathrm{p}}$ bonds are at the very long end of values expected for low-spin iron(II). ${ }^{15}$ The porphyrin core is planar; core diagrams for all three complexes are given in Figure S2.

Mössbauer spectra for $[\mathrm{Fe}(\mathrm{OEP})(\mathrm{CO})]$ are consistent with an isolated five-coordinate iron center. The quadrupole splitting is much larger $(1.84 \mathrm{~mm} / \mathrm{s}, 4.2 \mathrm{~K})$ than that observed for sixcoordinate carbonyls (typically less than $0.7 \mathrm{~mm} / \mathrm{s}$ ), strongly indicative of a d-orbital asymmetry consistent with five-coordination. The isomer shift value of $0.27 \mathrm{~mm} / \mathrm{s}$ is similar to that of the six-coordinate derivatives; the relatively low value is consistent with strong and effectively equivalent covalency among the occupied $\mathrm{Fe} 3 \mathrm{~d}$ orbitals in all derivatives. The sixcoordinate derivative is much more symmetric with a quadrupole splitting of 0 or near 0 at 4.2 $\mathrm{K}$ and isomer shift of $0.31 \mathrm{~mm} / \mathrm{s}$, this increases to a QS of $0.176 \mathrm{~mm} / \mathrm{s}$ and an isomer shift of $0.18 \mathrm{~mm} / \mathrm{s}$ at $298 \mathrm{~K}$. Spectra in applied magnetic field for this complex confirmed that it is a diamagnetic species. Complete data are in Table S1.

Five-coordinate coordinate carbonyl hemes have been structurally characterized and compared with related five- and six-coordinated diatomic complexes. Although the biological importance of five-coordinate carbonyl hemes is not known, proteins have been described that have spectroscopic properties that may be associated with this adduct. ${ }^{9,24}$ Additional spectroscopic and photophysical studies on these carbonyl complexes are in prospect.

\section{Supplementary Material}

Refer to Web version on PubMed Central for supplementary material.

\section{Acknowledgements}

We thank the NIH for support (GM-38401, W.R.S.) and the NSF for X-ray instrumentation (CHE-0443233). We thank Prof. Timothy Sage for useful discussions.

\section{References}

1. Gilles-Gonzalez MA, Gonzalez G. J Inorg Biochem 2005;99:1. [PubMed: 15598487]

2. Rodgers KR, Lukat-Rodgers GS. J Inorg Biochem 2005;99:963. [PubMed: 15811514]

3. Roberts GP, Kerby RL, Youn H, Conrad M. J Inorg Biochem 2005;99:280. [PubMed: 15598507](a) Aono S, Nakajima H. Coord Chem Rev 1999;190-192:267.(b)

4. Boon EM, Marletta MA. J Inorg Biochem 2005;99:892. [PubMed: 15811506]

5. The following abbreviations are used in this paper: Por, generalized porphyrin dianion; OEP, dianion of octaethylporphyrin; Deut, dianion of deuteroporphyrin; TPP, dianion of tetraphenylporphyrin.

6. Complete synthetic procedures are given in the Supplemental Information.

7. Rougee M, Brault D. Biochemistry 1975;73:4100.(a)Wayland BB, Mehne LF, Swartz J. J Am Chem Soc 1978;100:2379.(b) Strauss SH, Holm RH. Inorg Chem 1982;21:863.(c)

8. Sage, JT.; Champion, PM. Small Substrate Recognition in Heme Proteins. In: Suslick, KS., editor. Comprehensive Supramolecular ChemistryPergamon. 5. Oxford; UK: 1996. p. 171-217.

9. Pal B, Li Z, Ohta T, Takenaka S, Tsuyama S, Kitagawa T. J Inorg Biochem 2004;98:824. [PubMed: 15134928](a)Makino R, Obayashi E, Homma N, Shiro Y, Hori H. J Biol Chem 2003;278:11130. [PubMed: 12540839](b)

10. Silvernail NJ, Roth A, Schulz CE, Noll BC, Scheidt WR. J Am Chem Soc 2005;127:14422. [PubMed: 16218637]

11. Kim K, Ibers JA. J Am Chem Soc 1991;113:6077. 
12. Ellison MK, Scheidt WR. J Am Chem Soc 1997;119:7404.(a)Scheidt WR, Duval HF, Neal TJ, Ellison MK. J Am Chem Soc 2000;122:4651.(b)

13. Ellison MK, Schulz CE, Scheidt WR. Inorg Chem 2000;39:5102. [PubMed: 11233208]

14. Silvernail, N. J.; Noll, B.C.; Scheidt, W. R. unpublished results.

15. Scheidt, WR. Systematics of the Stereochemistry of Porphyrins and Metalloporphyrins. In: Kadish, KM.; Smith, K.; Guilard, R., editors. The Porphyrin Handbook . Academic Press; San Diego, CA and Burlington, MA: 2000. p. 3Chapter 16

16. Scheidt WR, Lee YJ, Hatano K. J Am Chem Soc 1984;106:3191.

17. Scheidt WR, Geiger DK. Inorg Chem 1982;21:2056.

18. Scheidt WR, Haller KJ, Fons M, Mashiko T, Reed CA. Biochemistry 1981;20:3653. [PubMed: 7260062]

19. Salzmann R, McMahon MT, Godbout N, Sanders LK, Wojdelski M, Oldfield E. J Am Chem Soc 1999;121:3818.

20. Silvernail, N. J.; Noll, B.C.; Scheidt, W. R. unpublished results.

21. Ellison MK, Scheidt WR. J Am Chem Soc 2001;123:5210.

22. Wyllie GRA, Schulz CE, Scheidt WR. Inorg Chem 2003;42:5722. [PubMed: 12950223]

23. Cao C, Dahal S, Shang M, Beatty AM, Hibbs W, Schulz CE, Scheidt WR. Inorg Chem 2003;42:5202. [PubMed: 12924891]

24. Chu GC, Katakura K, Tomita T, Zhang X, Sun D, Sato M, Sasahara M, Kayama T, Ikeda-Saito M, Yoshida T. J Biol Chem 2000;275:17494. [PubMed: 10751393](a)Vogel KM, Spiro TG, Shelver D, Thorsteinsson MV, Roberts GP. Biochemistry 1999;38:2679. [PubMed: 10052938](b) 


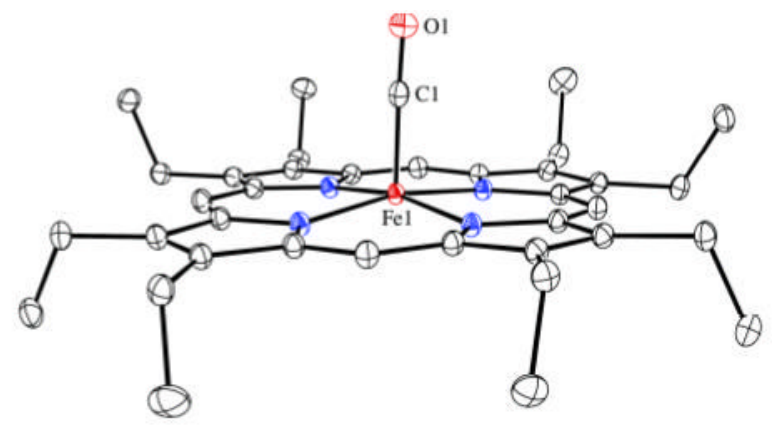

Figure 1.

Thermal ellipsoid plot of $[\mathrm{Fe}(\mathrm{OEP})(\mathrm{CO})]$ (50\% probability ellipsoids). Hydrogen atoms are omitted for clarity. 
a
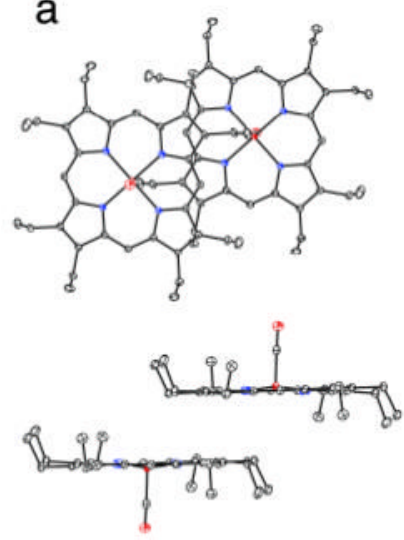

b
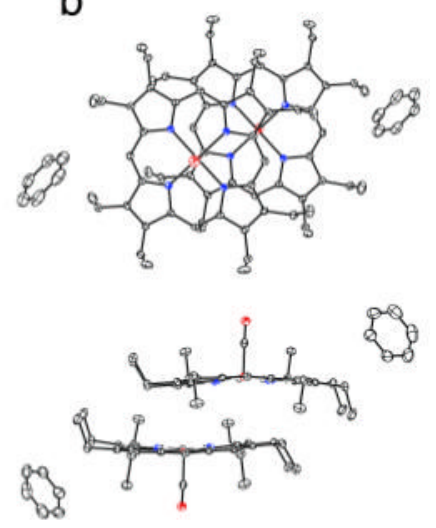

Figure 2.

ORTEP plots (50\% probability ellipsoids) of [ $\mathrm{Fe}(\mathrm{OEP})(\mathrm{CO})]$ (a) and $[\mathrm{Fe}(\mathrm{OEP})(\mathrm{CO})] \cdot \mathrm{C}_{6} \mathrm{H}_{6}$ (b) displaying the pairwise interactions. In (a) the 24-atom mean plane separation is $3.42 \AA$ and the lateral shift of the two ring centers is $6.76 \AA$. The $\mathrm{Fe}^{\cdots} \mathrm{Fe}$ distance is $7.58 \AA$. The corresponding distances in (b) are 3.46, 3.88, and $5.20 \AA$, respectively. 


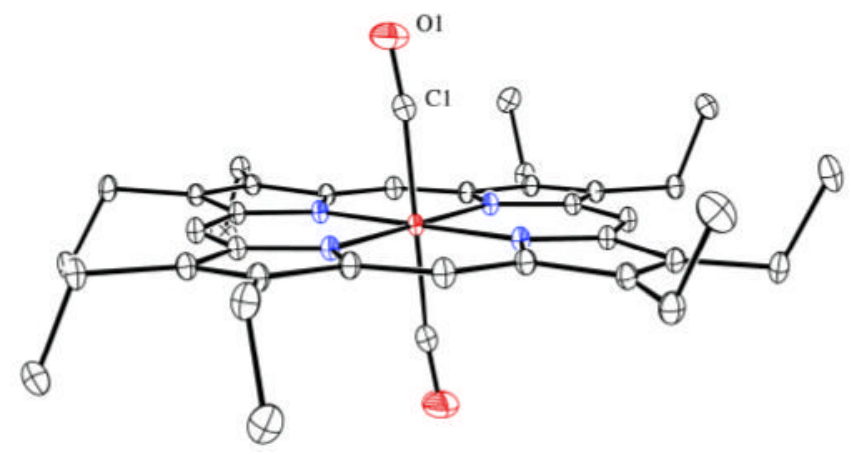

Figure 3.

Thermal ellipsoid plot of $\left[\mathrm{Fe}(\mathrm{OEP})(\mathrm{CO})_{2}\right]$. The $\mathrm{Fe}-\mathrm{C}$ vector is tilted from the heme normal by $5.9^{\circ}$; the $\mathrm{C}$ and $\mathrm{O}$ atoms are shifted laterally by 0.14 and $0.56 \AA$, respectively. 
data ns38 0m

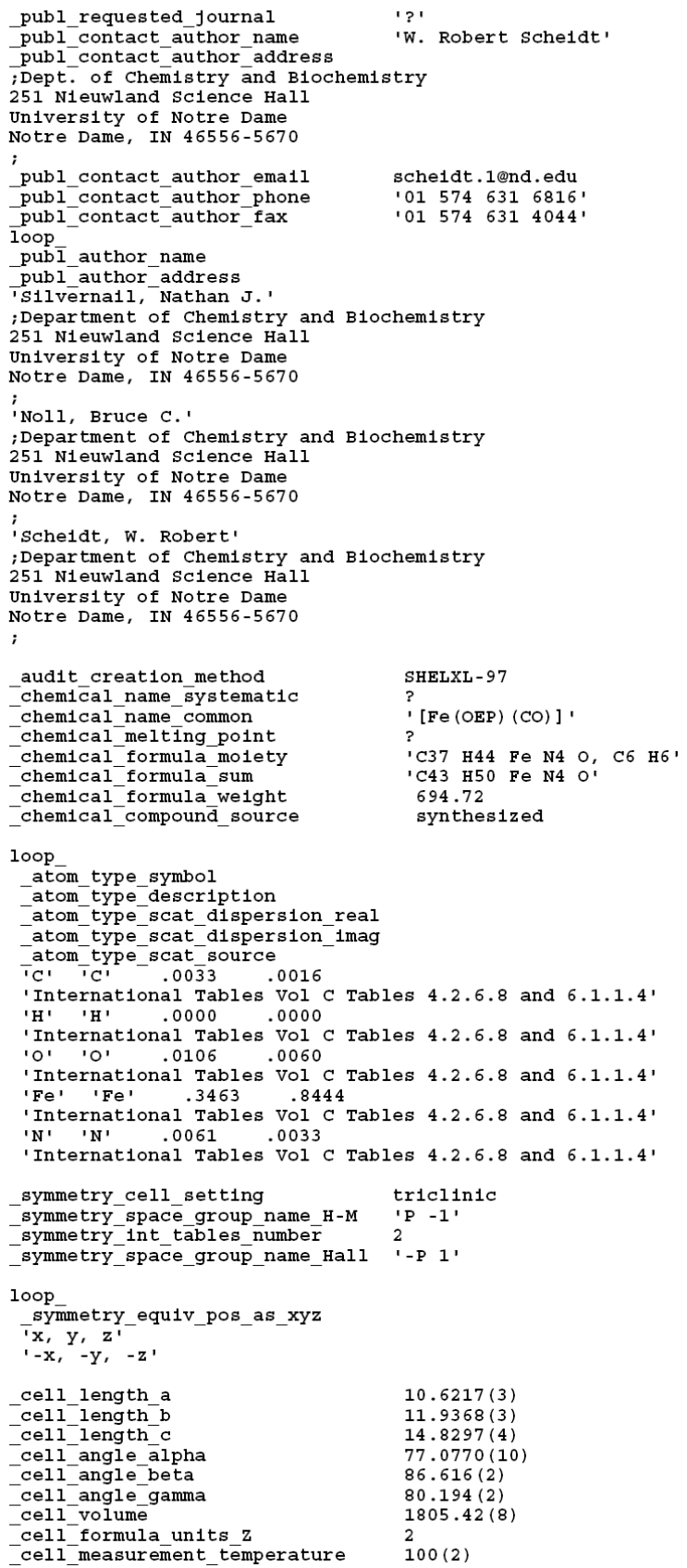




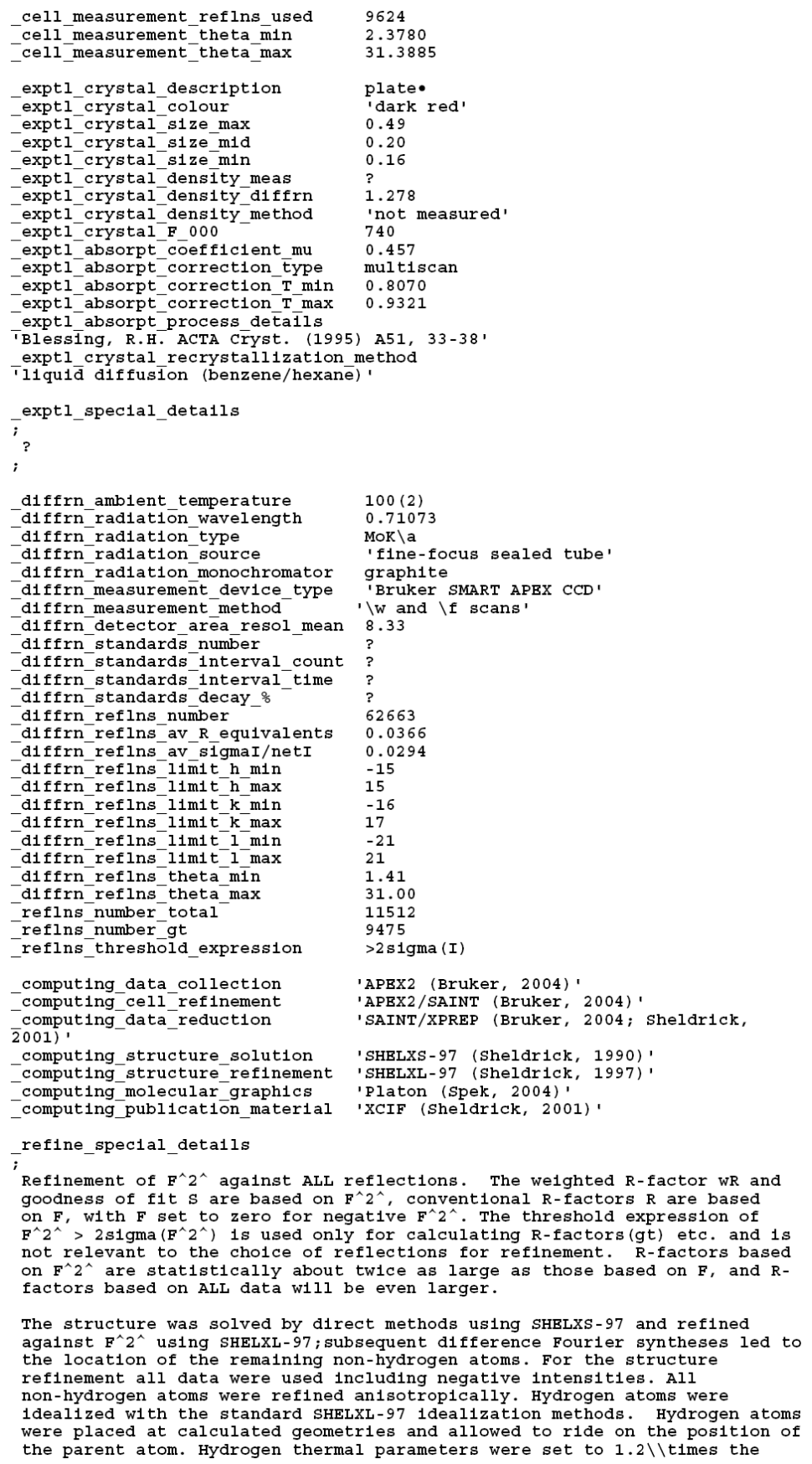


equivalent 1sotropic $U$ of the parent atom. The program SADABS was applied for the absorption correction.

The structure 1s a 1ron octaethylporphyrin bound axially carbon monoxide. $\begin{array}{ll}\text { refine_ls_structure_factor_coef } & \text { Fsqd } \\ \text {-refine_ls_matrix_type } & \text { full } \\ \text { refine_ls_welghting_scheme } & \text { calc }\end{array}$

refine 1 s welghting detalls
'calc $\mathrm{W}=1 /\left[\backslash \mathrm{s}^{\wedge} 2^{\wedge}\left(\mathrm{FO}^{\star} 2^{\wedge}\right)+(0.0371 \mathrm{P}){ }^{\wedge} 2^{\wedge}+0.9271 \mathrm{P}\right]$ where $\mathrm{P}=\left(\mathrm{FO}^{\wedge} 2^{\wedge}+2 \mathrm{FC}^{\wedge} 2^{\wedge}\right) / 3^{\prime}$ atom_sites_solution_primary direct -atom sites-solution secondary difmap atom sites solution hydrogens geom refine ls hydrogen treatment constr refine_ls_extinction_method none refine_ls_extinction_coef ?

refine_ls_number_refins 11512

refinels number restraints

-refine_ls_R_factor_all $\quad 0.0486$

refine_ls_R_factor_gt $\quad 0.0348$

refine_1s_WR_tactor_ref 0.0919

refine ls goodness of fit ref 1.045

refine_ls_restrained_s_ali 1.045

refine_ls_sh1ft/su_max $\quad 0.001$

refine ls shift/su mean 0.000

loop

atom_site_label

atom site type symbol

atom site fract $x$

atom site fract - y

atom site fract ${ }^{-}$.

atom site U 1so or equ1v

atom site adp type

atom site occupancy

atom_site_symmetry multiplicity

atom site calc flag

atom site refinement flags

atom_site disorder_assembly

atom_site disorder group

Fe1 Fe $0.37 \overline{7} 337(16) 0.350000$ (14) 0.455541 (11) 0.01184 (5) Uan1 $11 \mathrm{~d}$. . .

N1 N 0.44414 (9) $0.25436(9) \quad 0.36431(7) \quad 0.01241(18)$ Uan1 $11 \mathrm{~d} .$.

$\mathrm{N} 2 \mathrm{~N} 0.26846(9) \quad 0.45975(8) \quad 0.35922(7) \quad 0.01243(18)$ Uan1 $11 \mathrm{~d} . .$.

N3 $\mathrm{N} 0.33908(9) \quad 0.46522(8) \quad 0.53569(7) \quad 0.01255(18)$ Uan1 11 d . . .

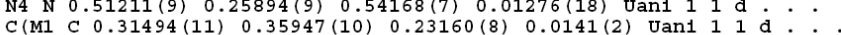

C(M1 C 0.31494 (11) $0.35947(10) \quad 0.23160$ (8) 0.0141 (2) Uan1 11 d . .

H (MA H $0.29490 .3603 \quad 0.1698 \quad 0.017$ U1so 11 calc $R$.

C(M2 C $0.16761(11) \quad 0.60134(10) \quad 0.44862(8) \quad 0.0140$ (2) Uan1 $11 \mathrm{~d} .$.

H(MB H 0.10120 .66530 .45000 .017 U150 11 CalC R.

C(M3 C $0.48818(11) \quad 0.37450(10) \quad 0.66007$ (8) 0.0142 (2) Uan1 $11 \mathrm{~d}$...

H (MC H $0.52020 .3817 \quad 0.7166 \quad 0.017$ U1so 1 l calc $R$.

C(M4 C $0.59338(11) \quad 0.10295(10) 0.46114(8) \quad 0.0135(2)$ Uan1 $11 \mathrm{~d}$. . .

H (MD H $0.64520 .02880 .4657 \quad 0.016$ U1so 11 calc R.

C(A1 C 0.53071 (11) $0.15239(10) \quad 0.37936(8) \quad 0.0125$ (2) Uan1 $11 \mathrm{~d}$. . .

C (A2 C $0.40580(11) \quad 0.26963(10) \quad 0.27474(8) \quad 0.0128$ (2) Uan1 11 d . . .

$C$ A C $0.25066(11) 0.44830(10) 0.27066(8) 0.0132(2)$ Jan1 11 d : .

$C\left(A 4 C_{1}\right.$ C 0.18141 (11) $0.55592(10) 0.37012$ (8) 0.0127 (2) Uan1 11 d . .

(A6 C $0.39181(11) 0.56029(10) 0.51927$ (8) 0.0134 (2) Uand 11 d.

C(A6 C C 0.39181 (11) 0.46029 (10) $0.61927(8)$ (8) 0.0134 (2) Uan1 11 d . . .

(A) $0.54156(11) 0.27918(10) 0.52505(8) 0.0134(2)$ Uan1 1.

C(A8 C 0.58619 (11) $0.15350(10)$

C B2 $0.47394(11) 0.17957(10) 0.23125(8) 0.0139(2)$ Uand 11 d .

C B2 C $0.47394(11) 0.17957(10) 0.23125(8)$. $0.0139(2)$ Uan1 11 d. .

C B4 C $0.11003(11) \quad 0.60644(10) 0.28702(8) 0.0138$ (2) Uant 11 d

C(B5 C $0.23544(11) \quad 0.61527(10) 0.60354(8)$ (8) 0.0141 (2) Uan1 11 d . .

C(B6 C $0.32926(11) 0.55353(10) 0.66172(8) 0.0139(2)$ Uant 11 d :

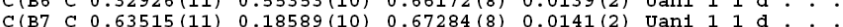

C B8 C $0.66104(11) 0.10607$ (10) $0.61890(8) 0.0135$ (2) Uant $11 \mathrm{~d}$.

C B8 C $0.66104(11)$.

H11A H $0.6147-0.03290 .23640 .020$ U1so 1 l calc $R$.

H11B H $0.6373-0.06300 .34540 .020$ U

C12 C $0.77913(13) 0.01917(12) 0.26990(11) \quad 0.0258$ (3) Uan1 $11 \mathrm{~d}$. . .

H12A H $0.8336-0.05210 .25990 .039$ U1sO 1 l CalC $R$.

$\mathrm{H} 12 \mathrm{~B}$ H $0.80910 .0419 \quad 0.32340 .039$ J1so $1 \mathrm{l}$ Calc $\mathrm{R}$.

$\begin{array}{llllllllll}\mathrm{H} 12 \mathrm{C} \text { H } & 0.7831 & 0.0818 & 0.2148 & 0.039 & \text { U1so } 1 \text { calc } R\end{array}$

C21 C $0.46465(13) 0.17777$ (11) $0.13144(8) 0.0188$ (2) Uant $11 \mathrm{~d}$.

H21A H $0.3735 \quad 0.1876 \quad 0.11590 .023$ U1so 1 l calc R. 


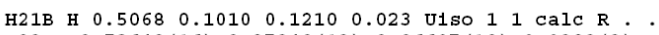

C22 C 0.52649 (16) 0.27340 (13) 0.06697 (10) 0.0282 (3) Uan1 11 d...

H22A H 0.51390 .27130 .00250 .042 O1s० 1 1 calc R..

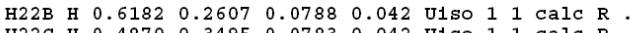

H22C H $0.4870 \quad 0.3495 \quad 0.0783 \quad 0.042$ U1so 1 l calc $R$.

C31 C $0.11401(12) 0.55857$ (12) $0.12695(8) 0.0192$ (2) Uan1 $11 \mathrm{~d}$. .

$\begin{array}{llllllll}\text { H31A H } & 0.0250 & 0.6003 & 0.1211 & 0.023 & 01 \text { so } 1 \text { l calc R. }\end{array}$

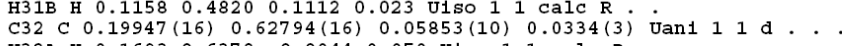

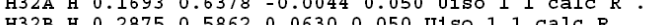

H32B H 0.28750 .58620 .06300 .050 U1so 1 1 calc $R$.

H32C H 0.19670 .70460 .07290 .05001 so 1 l calc $R$. .

H4 1A H $0.0067 \quad 0.7540 \quad 0.20990 .019$ U1so 11 calc R.

C42 C $-0.12585(12) \quad 0.67093(11) 0.29681(9) \quad 0.0200(2)$ Uan1 $11 \mathrm{~d}$...

$\mathrm{C} 42 \mathrm{C}-0.12585(12) \quad 0.67093(11) 0.29681(9) \quad 0.0200(2) \quad U a t$

H42A H -0.19250 .73990 .28930 .030 U1so 11 calc $R$.

H42 $\mathrm{H}-0.14090 .62230 .25460 .030 \mathrm{U} 1501$ 1 calc $\mathrm{R}$.

C51 C $0.13683(12) \quad 0.71548(10) \quad 0.61793(9) \quad 0.0170(2)$ Uant $11 d$.

H51A H $0.11430 .7680 \quad 0.5573 \quad 0.020$ U1so 11 calc R. .

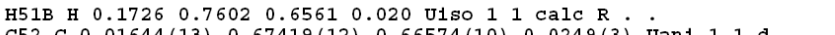

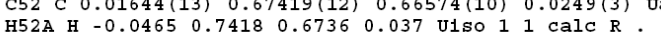

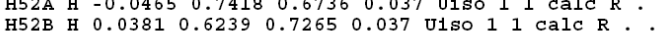

H52C H $0.0196 \quad 0.6304 \quad 0.6278 \quad 0.037$ U1so 1 1 Calc

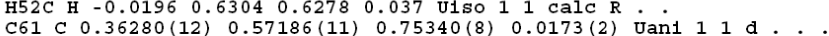

$\begin{array}{llllllll}\text { H61A H } & 0.3334 & 0.6544 & 0.7558 & 0.021 & \text { U1so } 1 & 1 & \text { calc R . }\end{array}$

C62 C 0.30452 (14) 0.49457 (13) $0.83620(9) 0.0241$ (3) Uan1 $11 d$.

$\begin{array}{llllllllll}\mathrm{H} 62 \mathrm{~A} \mathrm{H} & 0.3333 & 0.5085 & 0.8937 & 0.036 & \text { U1so } 1 & 1 & \mathrm{CalC} R\end{array}$

H62B H $0.3318 \quad 0.4126 \quad 0.83390 .036$ U1so 11 Calc $R$

H62 C H $0.21110 .51310 .8340 \quad 0.036$ U1so 1 l calc $R$.

C71 C 0.68766 (12) $0.18096(11) 0.76535(8) 0.0170(2)$ Uan1 $11 d . .$.

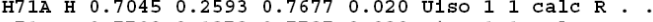

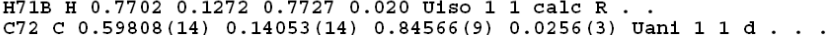

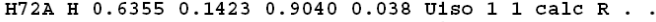

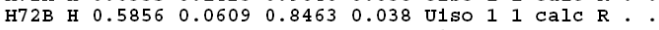

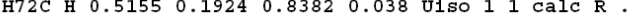

C81 C $0.75305(11)-0.00600(10) 0.63676(8) 0.0155(2)$ Uan $111 \mathrm{~d}$. .

H81A H $0.7189-0.06420 .6111 \quad 0.019$ U1so 1 1 calc R.

$\begin{array}{llllllllll}\text { H81B H } & 0.7611 & -0.0360 & 0.7044 & 0.019 & \text { U1so } 1 & 1 & \text { calc } R\end{array}$.

C82 C $0.88487(13) \quad 0.00863(12) 0.59375(11) 0.0262(3)$ Uan1 $11 \mathrm{~d}$..

H82A H $0.9429-0.06570 .60940 .039$ U1so 11 calc $R$.

$\begin{array}{llllllllll}\text { H82B H } & 0.9181 & 0.0676 & 0.6178 & 0.039 & \text { U1so } 1 & 1 & \text { calc } R \text {. }\end{array}$

$\begin{array}{lllllllll}\text { H82C H } & 0.8783 & 0.0334 & 0.5263 & 0.039 & \text { U1so } 1 & 1 & \text { calc } R\end{array}$.

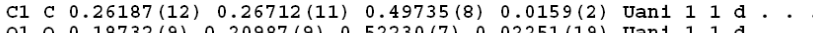

0100.18732 (9) 0.20987 (9) 0.52230 (7) 0.02251 (19) Uan1 $11 \mathrm{~d}$. .

C90 C $0.84363(16) 0.73398(16) \quad 0.98868(12) \quad 0.0359(4)$ Uan1 $11 \mathrm{~d}$. .

H9OA H 0.82860 .65571 .00610 .043 O1so 11 CalC R

C91 C $0.90715(16) 0.77079$ (18) $0.90674(13) 0.0399(4)$ Uan1 $11 \mathrm{~d}$...

H91A H 0.93630 .71820 .86760 .048 U1so 11 calc $R$.

C92 C 0.92837 (18) $0.88518(19) \quad 0.88154(14) 0.0469(5)$ Uan $111 \mathrm{~d}$.

$\mathrm{H} 2 \mathrm{~A} \mathrm{H} 0.9719(2) .9113 .0 .824$

C93 C $0.8863(2) 0.96126(19) 0.93885(16) 0.0560(6)$ Uan

H93A H 0.90131 .03950 .92200 .067 U1so 11 Calc $R$.

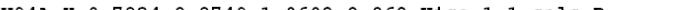

H9 A H 0.79240 .97491 .06020 .068 U1so 11 calc R

H95 H $0.7588 \quad 0.7829 \quad 1.1018 \quad 0.052$ U1so 1 l calc $R$.

loop

atom site aniso label

atom site aniso U 11

atom site aniso U 22

atom site an1so- ${ }^{-} 33$

atom_site_an1so_- ${ }_{-}^{-} 23$

atom_site_an1so_U_13

Fé1 $0.01199(8) 0 . \overline{0} 1 \overline{1} 81(8) \quad 0.01151(8)-0.00351(6)-0.00005(5)-0.00010(6)$

N1 $0.0116(4) \quad 0.0132(4) \quad 0.0122(4)-0.0030(3) \quad 0.0004(3)-0.0014(3)$

N2 $0.0124(4) \quad 0.0119(4) \quad 0.0127(4)-0.0028(3) \quad 0.0006(3)-0.0013(3)$

N3 $0.0129(4) 0.0123(4) 0.0124(4)-0.0032(3) 0.0005(3)-0.0014(3)$

N4 $0.0122(4) 0.0137(4) 0.0127(4)-0.0045(3) 0.0004(3)-0.0010(3)$

C (M1 0.0137 (5) $0.0162(5) \quad 0.0129(5)-0.0036(4)-0.0012(4)-0.0028(4)$

$\mathrm{C}(\mathrm{M} 20.0138(5) \quad 0.0116(5) \quad 0.0159(5)-0.0025(4) \quad 0.0019(4)-0.0014(4)$

C (M3 $0.0144(5) \quad 0.0153(5) \quad 0.0139(5)-0.0046(4) \quad 0.0004(4)-0.0035(4)$

C (M4 $0.0132(5) \quad 0.0113(5) \quad 0.0154(5)-0.0029(4) \quad 0.0008(4)-0.0006(4)$

C (A1 $0.0119(5) \quad 0.0124(5) \quad 0.0137(5) \quad-0.0039(4) \quad 0.0012(4)-0.0020(4)$

$\mathrm{C}(\mathrm{A} 20.0120(5) \quad 0.0141(5) \quad 0.0131(5)-0.0041(4) \quad 0.0007(4)-0.0032(4)$ 


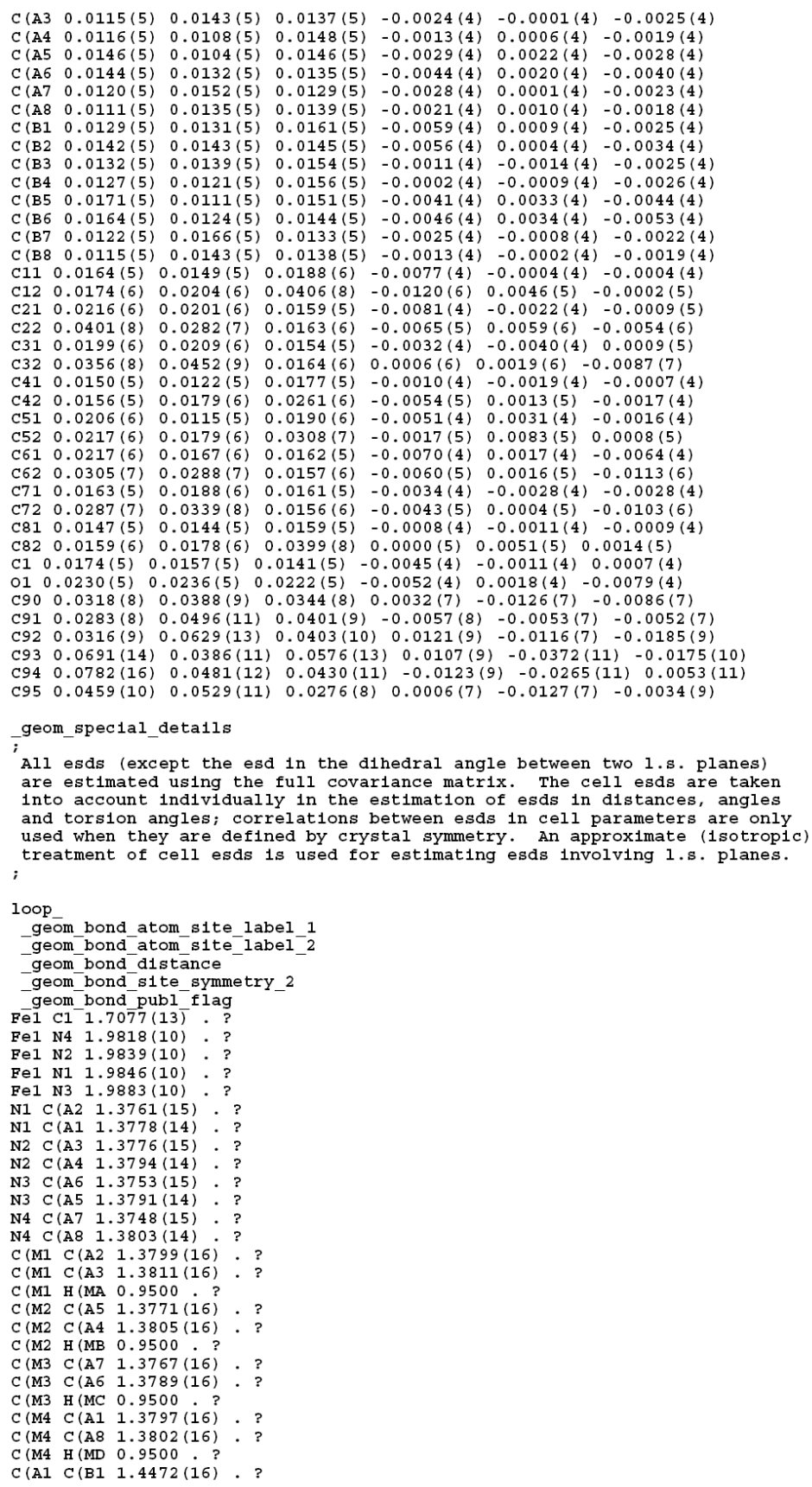




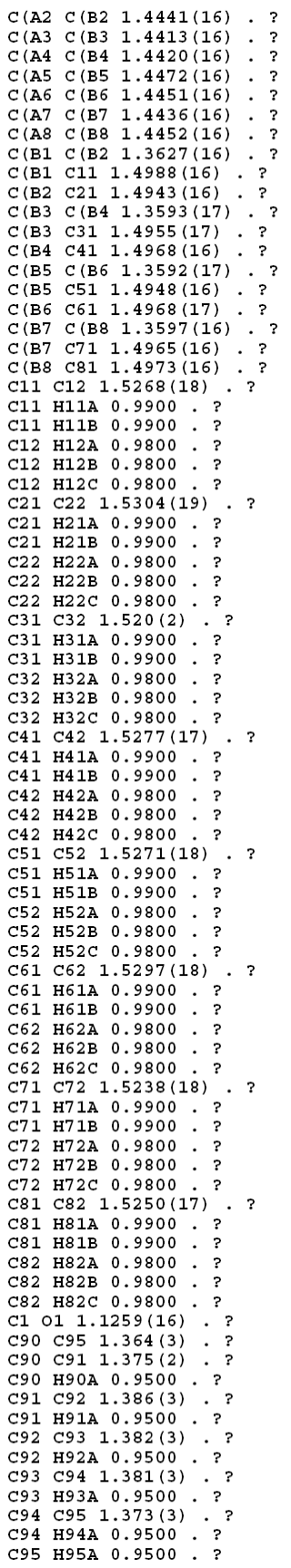

Inorg Chem. Author manuscript; available in PMC 2006 September 29. 
loop

_geom_angle_atom_site_label_1

label 2 geom_angle_atom_site_label geom_angle

_geom_angle_site_symmetry_1

geom_angle_site_symmetry

geom angle publ flag

C1 Fe1 N4 96.38(5) . . ?

C1 $\mathrm{Fe1}$ N2 $94.80(5) \cdot \cdot$ ?

C1 Fe1 N2 $168.82(4) \cdots$.

C1 Fel N1 93.31(5) * ? ?

Te1 N1 $89.99(4)$

C1 Fe1 N3 $98.76(5): \cdots$

44 Fe1 N3 $89.62(4) \cdots ?$

N2 Fe1 N3 $88.92(4) \cdot$

N1 Fe1 N3 $167.92(4)$

C(A2) 1 (A1 104 ?

C(A2 N1 Fe1 $127.49(8) \cdot{ }^{3}$

C (A2 N1 Fe1 $127.49(8)$

C(A1 N1 Fel $127.42(8)$. . ?

C A3 N2 C A4 104.70(9) . ?

C (A4 N2 Fe1 $127.27(8)$. ?

C (A6 N3 C C A5 104.49(9) ?

$\begin{array}{lllllll}C & (A 6 & \text { N3 } & \text { Fe1 } & 127.78(8) \\ C(A 5 & \text { N3 } & \text { Fel } & 127.34(8)\end{array}$

C (A7 N4 C (A8 104.60 (9) ??

C (A7 N4 Fe1 $127.59(8) \ldots$

C (A8 N4 Fe1 127.37 (8) . ?

$\mathrm{C}(\mathrm{A} 2 \mathrm{C}(\mathrm{M} 1 \mathrm{C}(\mathrm{A} 3 \mathrm{125.50}$ (11) . . ?

C (A2 C CM1 H(MA $117.3 \cdot \cdot ?$

C (A3 C CM1 H(MA 117.3 . $\cdot ?$

C (A5 C C (M2 C C (A4 124.70 (11)?

C (A5 5 C (M2 H

$\begin{array}{lllll}C(A 4 & \text { C (M2 } & \text { H(MB } & 117.7 & \text { : }\end{array}$ ?

C (A7 C (M3 C)(A6 124.97 (11)

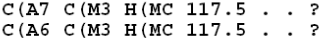

C (A1 C (M4 C(A8 124.69(11). . ?

C(A1 C (M4 H (MD 117.7 . ?

C (A8 C (M4 H(MD 117.7 . ?

N1 C CA1 C CM4 124.82(10) $:$ : ?

C ( 44 C C (A1 C C B1 124.15 (11): ? ?

N1 C (A2 C (M1 $124.67(10)$. : :

1 C (A2 C(B2 111.08 (10): .

C (MI1 C (A2 C C B2 124.24(11) . : ?

( $C$ (A3 $C(B 3$ )

C $\mathrm{CI} C\left(\mathrm{~A} 3 \mathrm{C}^{\mathrm{B} 3} 124.51(11) \cdot\right.$

N2 $C$ (A4 C (M2 124.76(11). ?

N2 C (A4 C (B4 111.11(10).

C (II2 C CA4 C (B4 124.08 (11)

C(N2 C (A5 N3 124.61(10) *

C(N12 C(A5 C(B5 124.21(11) - ?

N3 C (A5 C (B5 111.14(10) *

N3 C (A6 C(M3 124.66(11).

N3 $C(A 6 C)$ (B6 $111.48(10)$

$\mathrm{C}(\mathbb{1} 3 \mathrm{C}(\mathrm{A} 6 \mathrm{C}$ (B6 123.79 (11)

N4 C (A7 C (MB 125.08 (11) :

N4 C $($ A7 C (B7 111.14 (10) : ?

C ( M $\quad$ C (A7 C C B7 123.77 (11) . ?

C (M4 C (A8 N4 124.42(11). ?

C ( II C C (A8 C C B8 124.24 (11) . ?

N4 C (A8 C (B8 111.25 (10) . ?

C (B2 C (B1 C CA1 106.38(10) ? ?

C(B2 C (B1 C11 128.40(11) . ?

C(A1 C (B1 C11 125.16(11) : ?

C (B1 C (B2 C CA2 106.56(10) *? ?

C (B1 C (B2 C21 128.88(11) ..?

C (A2 C (B2 C21 124.46(11) *.?

C (B4 C (B3 C $(A 3$ 106.76(10) . ?

C(B4 C (B3 C31 127.33(11) . . ?

C (A3 C (B3 C31 $125.88(11)$

C (B3 C (B4 C(A4 106.42(10) •.

C(B3 C (B4 C41 127.59(11) :. ?

C(A4 C (B4 C41 125.94(11). * ?

C(B6 C (B5 C CA5 106.53(10) ․? $\begin{array}{llllll}C & \text { (B6 } & \text { C (B5 } & \text { C5 } 1 ~ & 128.04(11) & :\end{array}$ 


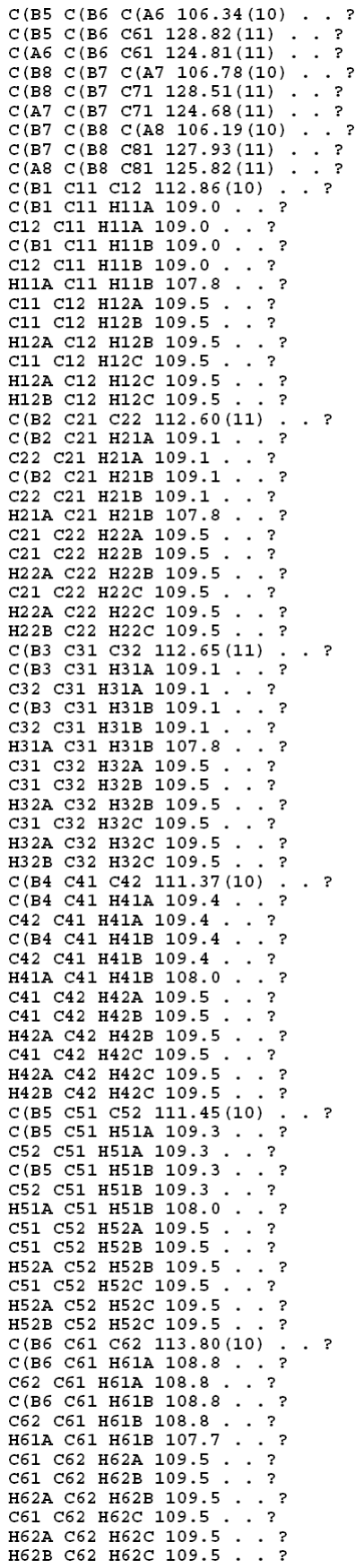

Inorg Chem. Author manuscript; available in PMC 2006 September 29. 


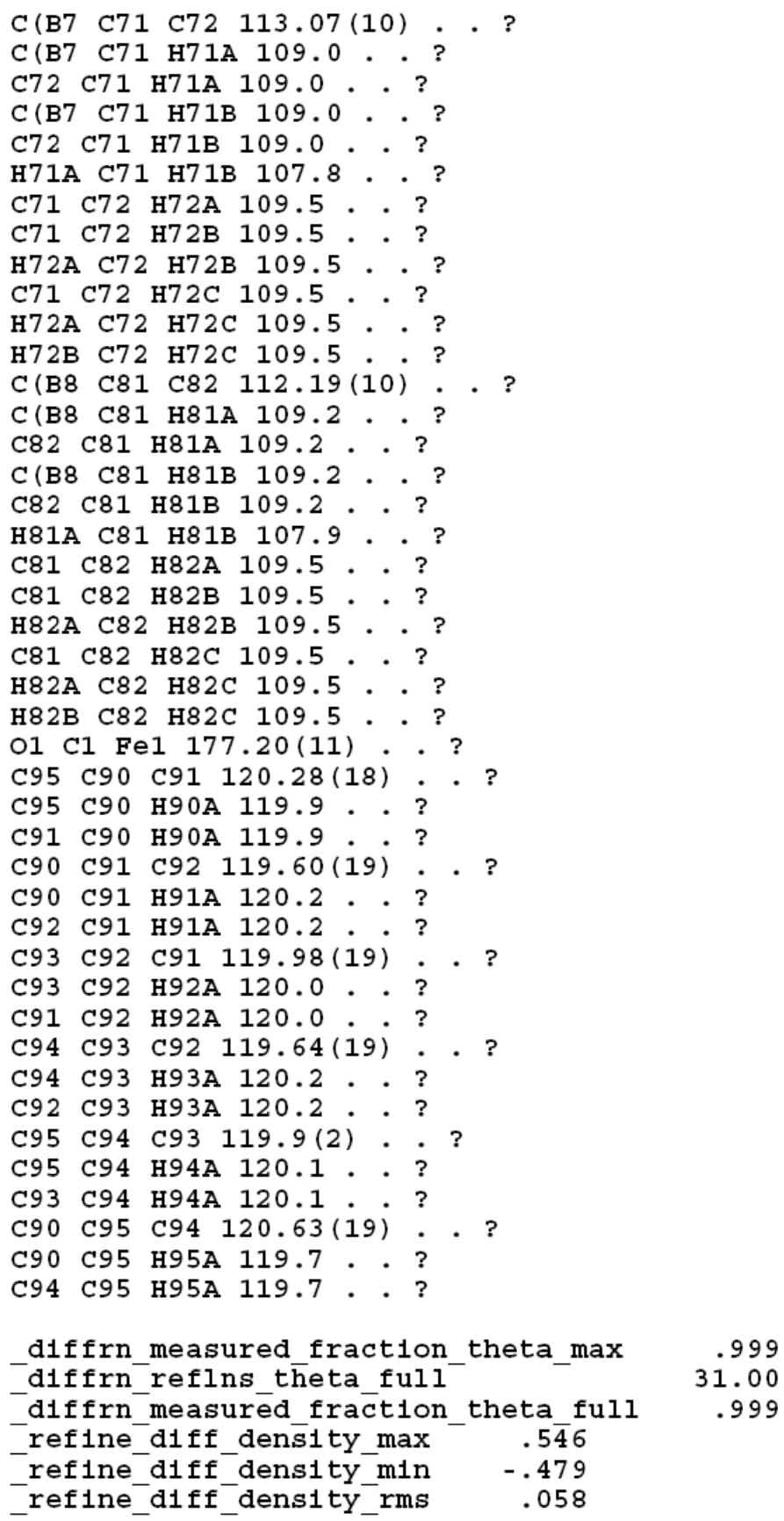


Table 1

Notable bonding parameters for $[\mathrm{Fe}(\mathrm{OEP})(\mathrm{CO})]$ and related compounds.

\begin{tabular}{|c|c|c|c|c|c|c|c|c|}
\hline Complex & $\mathrm{Fe}-\mathrm{XY} \mathrm{Y}^{a}$ & $\mathrm{X}-\mathrm{Y}^{a}$ & $\mathrm{Fe}-\mathrm{X}-\mathrm{Y}^{b}$ & $\mathrm{Fe}-\mathrm{Np}{ }^{a}$ & $\mathrm{Fe}-\mathrm{L}_{\mathrm{ax}} a$ & $\Delta \mathrm{Fe}^{d}$ & $v \mathrm{X}-\mathrm{Y}^{c}$ & ref \\
\hline$[\mathrm{Fe}(\mathrm{OEP})(\mathrm{CO})]$ & $\begin{array}{l}1.7140 \\
(11)\end{array}$ & $\begin{array}{l}1.1463 \\
(12)\end{array}$ & $177.20(8)$ & $1.988(2)$ & - & 0.20 & $1944^{e}$ & tw \\
\hline $\begin{array}{l}{[\mathrm{Fe}(\mathrm{OEP})} \\
(\mathrm{CO})] \cdot \mathrm{C}_{6} \mathrm{H}_{6}\end{array}$ & $\begin{array}{l}1.7077 \\
(13)\end{array}$ & $\begin{array}{l}1.1259 \\
(16)\end{array}$ & $\begin{array}{l}177.20 \\
(11)\end{array}$ & $1.984(3)$ & - & 0.22 & $1948^{e}$ & tw \\
\hline$[\mathrm{Fe}(\mathrm{OEP})(\mathrm{NO})]$ & $1.722(2)$ & $1.167(3)$ & $144.4(2)$ & $2.004(15)$ & - & 0.29 & $1666^{e}$ & 12 \\
\hline$[\mathrm{Fe}(\mathrm{OEP})(\mathrm{NO})]$ & $1.7307(7)$ & $\begin{array}{l}1.1677 \\
(11)\end{array}$ & $142.74(8)$ & $2.010(13)$ & - & 0.27 & $1673^{e}$ & 12 \\
\hline$[\mathrm{Fe}(\mathrm{OEP})(\mathrm{NO})]^{+}$ & $\begin{array}{l}1.6528 \\
(13)\end{array}$ & $1.140(2)$ & $\begin{array}{l}173.19 \\
(13)\end{array}$ & $1.994(5)$ & - & 0.32 & $1838^{e}$ & 13 \\
\hline$[\mathrm{Fe}(\mathrm{OEP})(\mathrm{NO})]^{+}$ & $1.644(3)$ & $1.112(4)$ & $176.9(3)$ & $1.994(1)$ & - & 0.29 & $1868^{e}$ & 16 \\
\hline$[\mathrm{Fe}(\mathrm{OEP})(\mathrm{CS})]$ & $1.662(3)$ & $1.559(3)$ & $176.3(2)$ & $1.982(5)$ & - & 0.23 & $1292^{e}$ & 17 \\
\hline $\begin{array}{l}{[\mathrm{Fe}(\text { Deut })(\mathrm{CO})} \\
(\mathrm{THF})]\end{array}$ & $1.706(5)$ & $1.144(5)$ & $178.3(14)$ & $1.98(3)$ & $2.127(4)$ & 0.10 & $1955^{f}$ & 18 \\
\hline $\begin{array}{l}{[\mathrm{Fe}(\mathrm{OEP})(\mathrm{CO})} \\
(1-\mathrm{MeIm})]\end{array}$ & $1.744(5)$ & $1.158(5)$ & $175.1(4)$ & $2.000(3)$ & $2.077(3)$ & 0.00 & $1965^{g}$ & 19 \\
\hline $\begin{array}{l}{[\mathrm{Fe}(\mathrm{OEP})(\mathrm{CO})} \\
(1-\mathrm{MeIm})]\end{array}$ & $\begin{array}{l}1.7733 \\
(12)\end{array}$ & $\begin{array}{l}1.1413 \\
(15)\end{array}$ & $\begin{array}{l}175.67 \\
(11)\end{array}$ & $2.010(4)$ & $\begin{array}{l}2.0544 \\
(9)\end{array}$ & 0.02 & $1980^{e}$ & 20 \\
\hline $\begin{array}{l}{[\mathrm{Fe}(\mathrm{OEP})(\mathrm{NO})} \\
(1-\mathrm{MeIm})]^{+}\end{array}$ & $\begin{array}{l}1.6465 \\
(17)\end{array}$ & $1.135(2)$ & $\begin{array}{l}177.28 \\
(17)\end{array}$ & $2.003(5)$ & $\begin{array}{l}1.9889 \\
(16)\end{array}$ & 0.02 & $1921^{e}$ & 21 \\
\hline $\begin{array}{l}\text { [Fe(TPP)(NO) } \\
(1-\mathrm{MeIm})]\end{array}$ & $1.750(2)$ & $1.182(3)$ & $137.7(2)$ & $2.008(13)$ & $2.173(2)$ & 0.04 & $1628^{e}$ & 22 \\
\hline $\begin{array}{l}{[\mathrm{Fe}(\mathrm{OEP})(\mathrm{CS})(1-} \\
\mathrm{MeIm})]\end{array}$ & $1.703(4)$ & $1.563(4)$ & $172.2(2)$ & $2.001(4)$ & $2.112(3)$ & 0.10 & $1272^{e}$ & 23 \\
\hline$\left[\mathrm{Fe}(\mathrm{OEP})(\mathrm{CO})_{2}\right]$ & $\begin{array}{l}1.8558 \\
(10)\end{array}$ & $\begin{array}{l}1.1216 \\
(13)\end{array}$ & $173.95(9)$ & $2.0133(7)$ & $\begin{array}{l}1.558 \\
(10)\end{array}$ & 0.00 & $2021^{e}$ & tw \\
\hline
\end{tabular}

$a_{\AA}$

$b_{\text {degrees. }}$

${ }^{c}{ }_{\mathrm{cm}-1}$

${ }^{d}$ Displacement from 24-atom meanplane.

${ }^{e}$ Nujol mull.

$f_{\text {THF. }}$

${ }^{g} \mathrm{CD}_{2} \mathrm{Cl}_{2}$ solution. 